G. C. BOLTEN.

Of these 182 cases in 57 the onset of epilepsy was before the fifth year, in $3^{\circ}$ the onset was between the fifth and the tenth year, $3^{I}$ between the tenth and fifteenth year, I9 between the fifteenth and twentieth year, leaving only 45 cases in whom the age at onset was 20 or over. Four of the syphilitic cases are in the latter group.

In the ordinary course of events syphilis is not particularly apt to be acquired before the twentieth year, but on the other hand about three fourths of the cases of epilepsy have their onset before this time. According to this study it would seem a fair assumption; that of the epilepsies beginning after the twentieth year we may expect about 4 per cent. to be of syphilitic origin.

In regard to treatment of these cases the serum reaction of one was made negative by intravenous injection of salvarsan, another had four injections and mercury by inunction when he left the hospital with his serum still positive. One left the hospital within a few days after admission after receiving a few inunctions of mercury, two are still under treatment.

\title{
RESEARCHES ON THE PATHOGENESIS OF GENUINE EPILEPSY
}

BY

DR. G. C. BOLTEN, the Hague (Holland).

As the pathogenesis of genuine (idiopathic or primary) epilepsy is quite unknown until this moment, I have limited myself in as far as possible, in my researches and experiments, to sufferers of so-called genuine epilepsy and as this diagnosis until now is only to be made "per exclusionem" (therefore we must exclude as well as possible the countless forms of cerebral epilepsy and also "epilepsia tarda"; consequently I had at the outset to rely almost 
RESEARCHES ON THE PATHOGENESIS OF GENUINE EPILEPSY. 3OI

exclusively on anamnestic information), it was not always possible to exclude errors in the diagnosis. These errors were as far as possible proved, unless the sufferer had patience enough for a very prolonged and systematic treatment.

By means of a large clinical material of sufferers of genuine epilepsy, I tried to discover if this disease might have been originated by:

a) intestinal putrifaction (abnormal fermentations in the stomach or in the intestinal tract), intestinal parasites, chronic constipation or other intestinal derangements, such as chronic enteritis, or disturbances of the fermentation in the stomach.

b) intoxication by the purinic bases, by the albumoses or by other breaking-products of albumines. Also the possibility of common-saltretention and secondary salt-intoxication has been inquired into.

c) troubles in the function (hypersecretion or hyposecretion) of one or more glands with internal secretion (hypophysis, thymus, thyroid, parathyroids, pancreas, liver, spleen, ovarium, testicle and adrenals; experiments with the epiphysis could not be performed).

All these researches and experiments have taken nearly ten years and have always been conducted for several patients at one time and nearly always on clinical sufferers, who consequently were under continual and good observation.

The results of these experiments and their deductions may be resumed as follows:

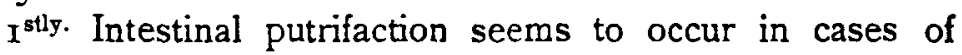
epilepsy only as a great exception and when this phenomenon exists, there is produced in the intestinal tract an abnormal quantity of indol, skatol and phenol, which appear in the urine as indican. Therefore, in case of intestinal putrifaction, one finds an increased standard of indican of the urine. In a great number of epileptics we have 
determined the indican-standard, but important increases never were found; nor was aceton discovered in the urine of epileptics.

For these reasons it must be an evident fact, that intestinal putrifaction does not play a part in genuine epilepsy. And also one does not find chronic diarrhoeas in epileptics, unless due to faulty dieting, while intestinal parasites as well as other intestinal troubles, as chronic constipation, also have no influence of any importance upon the fits and the other epileptic phenomena.

Moreover, Moore Alexander controlled exactly the intestinal flora of epileptics and compared it with that of nor mal persons, without finding any remarkable difference.

The great influence, which many investigators (Mac Caskey, Spratling, Bouman, de Fleury, Rodiet) ascribe to stomachal and intestinal troubles does not exist at all. And the atonic and dilated stomach (Mangelsdorff, de Groot) whether accompanied or not by hyperchlorhydria belongs to the secondary alterations, such as are found a great many in epileptics and which, concerning pathogenesis, do not count at all.

The administration of an extremely easily digestible diet, the regular use of intestinal desinfectants, the removal of intestinal parasites, the continual cleaning of the intestinal canal, the careful prevention of chronic constipation, all that, in short, has only slight influence on the phenomena of the chronic intoxication, which we call genuine epilepsy.

Much attention was paid by us to the part, played by the feeding-albumines and their breaking-products (purinic bases, albumoses), but the results also were purely negative. It is realey not quite clear, why many investigators have ascribed such an important position to the purinic bases, because all those substances (xanthine, paraxanthine, guanine, karnine, hypoxanthine, adenine etc.; caffeine and theobromine also beiong to them) are fairly harmless and are changed (except caffeine and theobromine) by oxida- 
tion into uric acid. If the purinic bases should be insufficiently oxidized and excreted, then there would be formed in case of epilepsy less exogene uric acid than in normal persons, who keep quite the same diet. Concerning this there was no evidence: epileptics (cerebral as well as genuine epilepsy) show, as we could demonstrate by ample researches, quite the same standard of uric acid of the urine as normal persons, who have been kept on quite the same diet and who live under entirely the same conditions.

And moreover, the purinic bases are not or but very little poisonous; adenine is the most poisonous and yet we can give adenine to epileptics with safety: they do not react to it in any way. The food, rich with purines, does not bring any change in the phenomena and even when we put the patient for a considerable period on a quite "purine-free" diet (which is very well possible and can be continued for a long time), we do not get a marked improvement. Investigators, who thought to have obtained by the purine-free diet an important improvement have not continued their experiments long enough or else they have experimented on too few patients. Hoppe also did not observe the slightest difference between the phenomena, in either highly-purinic or purinefree foods, carried as far as possible.

And when we retain all albumines, animal as well as vegetable (which however cannot be carried through perfectly), we also do not notice an improvement of any im portance. So it is impossible to ascribe any part to the albumoses, which are, as is well known, very poisonous (and which, therefore, are transformed synthetically into plasma-albumine during the passage through the intestinal well); genuine epilepsy thus is not an intoxication by albumoses or by other breaking-products of albumines.

In the eventual ordinary salt-retention and secondary intoxication we find quite the same: when we eliminate as far as possible all ordinary salt from the food (the diet poor 
of salt), this has not the slightest influence on the fits or on the other phenomena, unless we apply bromides (moderate dosis) at the same time, and only by this, namely the application of bromides in the diet poor of salt, the favourable results proceed, which many investigators (Bouman, Bailint, Lortat-Jacob, Roux, Gordon) obtained with the treatment of Toulouse and Richet; the diet poor of salt, without simultaneous bromide-application is completely inactive, as I could establish in a great number of patients; van der Kolk found the same. The treatment according to Toulouse and Richet simply depends upon this, that by the abstinence of ordinary salt and the simultaneous application of bromides, the latter accumulates much more rapidly in the organism than when combinig it with the use of salt; thereby a much larger bromideaccumulation ("Bromide-depot", Hoppe, Laudenheimer) is formed and thus the sedative effect of the bromide salts is much more intense, nothing more.

Also a pure milk-diet, by which Bergmann asserts to have obtained good results, has no more favourable influence on the fits or on the other epileptic phenomena.

In general we can conclude, as is proved also by our experiments, first that all kinds of intestinal and stomachal troubles exercise a slightly infavourable influence on the epileptic phenomena, and secondly, that in general there does not exist a single diet, that in the long run really exercises any noticeable influence on the kind, the intensity and the quantity of the epileptic "discharge" (the fit) or on the other phenomena.

A great many and lasting experiments have been made by us in our endeavour to determine, if one or more of the glands with internal secretion have any influence on the origin or on the different phenomena of the epileptic disease. To this must, of course, be considered the possibility of an intoxication by an eventual hyperfunction of one of those organs, the more so, because Volland, Ohlmacher and others often found a persistent thymus, 
and other congenital alterations ("status thymo-lymphaticus", narrowed aorta etc.) in their pathological-anatomical researches in epileptics. But neither by the application of extract of fresh thymus, nor by that of one of the other glands with internal secretion, could any decrease of the phenomena be caused, not even when large quantities were applied. (I wish to remark, that in my experiments always fresh press-extracts of the glands with internal secretion were used and that these fluids were always given by the rectal way, in order to prevent their eventual destruction by the gastric juice. Dry organs, given in tablet-form are quite useless, because they contain, as appears by their smell, breaking-products and therefore they cannot in any way imitate the physiological function of the organs; moreover most of these glands contain ferments, which are destroyed by the dryingprocess).

A long series of experiments has been made with the press-extracts of nearly all glands with internal secretion (hypophysis, thyroid, parathyroids, liver, pancreas, adrenals, ovarium, testicle, thymus and spleen); their results can be capitulated as follows:

I sty, that by the application of none of the pressextracts of these glands could an increase of one or more of the epileptic phenomena be obtained and that, consequently, genuine epilepsy surely does not depend upon a hyperfunction of one of the above mentioned glands with internal secretion, and

$2^{\text {ntly, that }}$ on the contrary we obtained a slight improvement in the sufferers of genuine epilepsy (the diagnosis, for want of better, taken "per exclusionem") by administering press-extracts of the parathyroids, and a very remarkable improvement by the rectal application of the combined press-extracts of the thyroid and parathyroids A great many cases of cerebral epilepsy (the diagnosis taken also chiefly from anamnestic information, or cases with phenomena of primary cerebral affections) almost failed to react in any way upon this treatment. 
It cannot be denied, that my results were very positive; they were too constant and too lasting, to justify any doubt; indeed amongst my patients there are:

two, who formerly had numerous fits (during respectively 4 and 8 years) and who now already are quite free from all phenomena for more then three years;

ten, who are already free from epileptic symptoms during 8 to 12 months; and

ten others, who have not exhibited the slightest phenomenon during 3 to 4 months, although before this new treatment they all had regularly 4-io fits a month.

With this treatment all application of bromides was immediately excluded, and so we can conclude from my experiments, with confidence, that genuine epilepsy is a chronic auto-intoxication depending upon the hypofunction of the thyroid and parathyroids.

In what manner are we to consider the pathogenesis of genuine epilepsy?

According to new researches of many biologists and physiologists (Hirsch, Biedl, Falta, Eppinger, Magnus Levy, Halliburton, Oswald, Rothschild and Lévi) the thyroid gland has several very complicate, important and divergent functions. Apart from the predominating part it plays, in association with the hypophysis and the thymus, on the development of the young organism, it has an accelerating influence on metabolism in general; the salt - as well as the nitrogen - and the phosphor-metabolism are mainly under its influence. Consequently, when the thyroids function fails, an important diminution of the excretion of phosphor-, calcium-, and magnesiumsalts, as well in defecation as in urine, appears, while the gas-change in the lungs also is greatly diminished.

Moreover, the action of the chromaffine-system and of the adrenaline becomes less intense by hypothyroidism; the adrenals and the thyroid supplement each others function 
and both have to support a tonus in the sympathetic system, in opposition to the pancreas, which has a retarding influence on it (Caro, Biedl). And so we can say, that the thyroid gland and the sympathetic nerve form more or less a closed system: the thyroid is innervated by the ganglion inferius of the sympathetic nerve (Steiner: according to Asher and Flach it also receives branches from the $n$. laryngeus sup. and from the $n$. laryngeus inf.) and on the other side the thyroid supports a tonus in the sympathetic system; consequently after thyroidectomy a diminished sympathetic-function appears (retarding intestinal peristaltic, lessened secretion of intestinal ferments, trophicand circulatory-troubles of the skin etc.).

Other investigators (Juschtschenko, Léopold Lévi and Rothschild) have accomplished these experiments and determined, that the thyroid accelerates the formation of all kinds of ferments of the intestinal tract and of the intermediary metabolism. In consequence of hypothyroidism a remarkable quantitative decrease of all these ferments appears; Juschtschenko has determined this especially concerning nuclease, katalase, lipase und peroxydase; phylokatalase, on the contrary, is secreted in increased quantity after thyroidectomy.

Further, according to Fassies, the thyroid accelerates the formation of alexines in the blood and increases the antibactericid action of the blood, whilst Walter has determined, that in thyroidectomised animals the regeneration of injured nerves took place much slower than in normal anjmals. It is not yet proved, whether the thyroid has a directly depoisoning action on blood (Trendelenburg).

Concerning the physiological function of the parathyroids, there is known less still with sufficient certainty; some investigators believe, that after parathyroidectomy the quantity of ammonia in the blood increases; however the acidosis found by Morel, von Fürth and others, is prob. ably not a direct consequence of the hypoparathyroidism, but of a phenomenon of the parathyroprive tetany, espe- 
308 G. C. BOLTEN, RESEARCHES OF THE PATHOGENESIS USW.

cially of the numerous and violent convulsions: the increase of the blood-ammonia in this case is a reaction of the organism against the lactic acid, formed by the musclecontractions.

To understand the phenomena of genuine epilepsy, we still need the results of the very important researches of Guillain and Laroche, who were able to show experimentally, that the cerebral cortex has a great affinity to different poisons as the diphteria- and the tetanus-toxin, and many others, as alcohol, aether, different alcaloids (morphine, cocaïne, absinthe) and other poisons, as tuberculine etc.

In the case of genuine epilepsy there exists hypothyroidism and hypoparathyroidism; consequently metabolism is retarded and less complete, because all kinds of ferments (as well of the intestinal tract as of the intermediary metabolism), indispensable for metabolism, are insufficiently produced, and all kinds of toxic products of our own metabolism as well as breaking-products of feedingmaterials come into the circulation and gradually they accumulate and become lodged in the cerebral cortex, which, when this accumulation of toxins has reached its maximum, react upon that with a so-called "discharge", the epileptic fit, as a temporary remedy of the organism to deliver itself of the toxins for a short period; thus a fit is indeed a defending-reaction of the organism against the accumulation of toxins.

Our experiments and their results lead to the following deductions and conclusions:

I $^{\text {sty. }}$ Of all cases of epilepsy, which make their appearance quite as genuine (idiopathic) epilepsy, an important part, depending upon divergent, primary cerebral affections, belongs to the cerebral (symptomatic) epilepsy and but the smaller part to the genuine epilepsy.

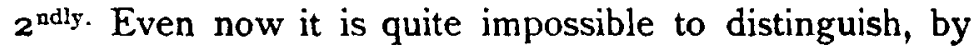
means of purely clinical phenomena, most cases of cerebral epilepsy (namely the numerous cases, originated by 
ANATOMIE, ANATOMIE PATHOLOGIQUE ET hiStOLOGIE. 309

a chronic, diffuse meningo-encephalitis) from genuine epilepsy.

The differential-diagnosis between these quite divergent affections must lie in the sphere of the pathology of metabolism.

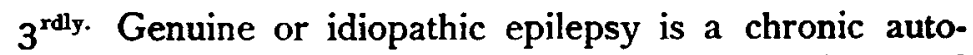
intoxication, caused by a hypofermentation of the intestinal tract and of the intermediary metabolism as a consequence of hypothyroidism and hypoparathyroidism (which is perhaps caused by troubles in the nervous elements of the thyroid and parathyroid glands, viz. the sympathetic nerve or its centres).

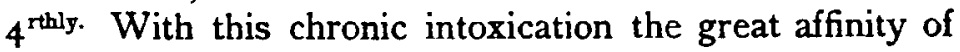
the cerebral cortex to a great many and very divergent poisons, is acting an important part.

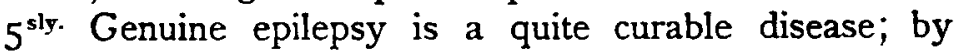
the administration, rectally, of fresh press-extracts of the insufficient organs (thyroid and parathyroid glands), one succeed, to free the patients from all epileptic phenomena, as I have proved in a great series of cases.

\section{ANALYSE DES TRAVAUX ORIGINAUX.}

\section{I. - ANATOMIE, ANATOMIE PATHOLOGIQUE ET HISTO- LOGIE.}

RUBY L. CUnNINGHaM, Berkley, Cal. The scaphoid SCAPULA: A NORMAL variation in MAN. Arch. Int. Med. X, p. $5^{89}$.

Graves of St. Louis reported some time ago, in a series of papers, upon the significance of the scaphoid scapula as a sign of degeneracy. Cunningham discusses this subject and presents a considerable amount of anthropometric data to show that the scaphoid scapula is a normal variation, quite as common among normals as among deviates.

J. F. Munson, Sonyea. 